\title{
Cover Page
}

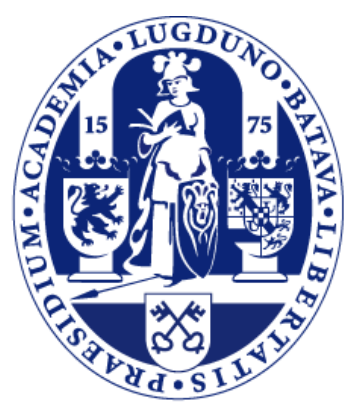

\section{Universiteit Leiden}

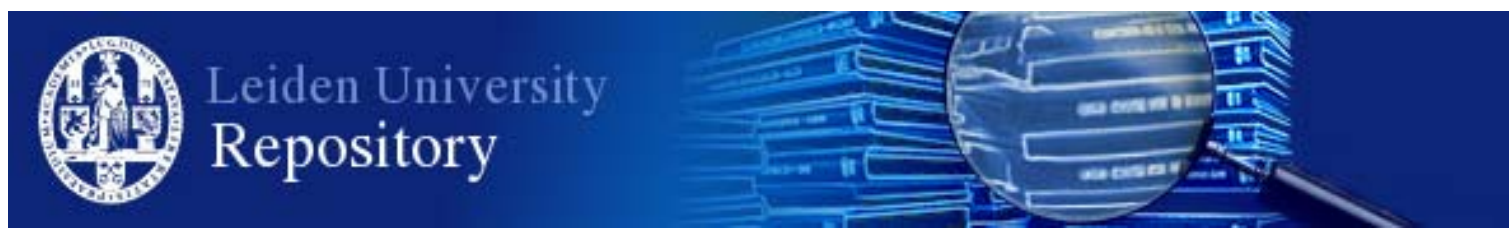

The handle http://hdl.handle.net/1887/19855 holds various files of this Leiden University dissertation.

Author: Solingen, Coen van

Title: The role of microRNA-126 in vascular homeostasis

Date: 2012-09-26 
CHAPTER 3

\section{Antagomir-mediated silencing of endothelial cell specific microRNA- I 26 impairs ischemia-induced angiogenesis}

Coen van Solingen', Leonard Seghers ${ }^{2,4}$, Roel Bijkerk', Jacques M.G.J. Duijs', Marko K. Roeten', Annemarie M. van Oeveren-Rietdijk', Hans. J. Baelde', Matthieu Monge', Joost B.Vos', Hetty C. de Boer', Paul H.A. Quax ${ }^{2,4}$,

Ton J. Rabelink' and Anton Jan van Zonneveld'

'Department of Nephrology and the Einthoven Laboratory for Experimental Vascular Medicine, ' 2 Department of Surgery and ${ }^{3}$ Department of Pathology, LUMC, Leiden, The Netherlands, ${ }^{4}$ Gaubius laboratory, TNO Biosciences, Leiden, The Netherlands.

J Cell Mol Med. 2009 Aug; I3(8A):1577-85. 


\section{Abstract}

MicroRNAs are negative regulators of gene expression that play a key role in cell-type specific differentiation and modulation of cell function and have been proposed to be involved in neovascularization. Previously, using an extensive cloning and sequencing approach, we identified miR-I26 to be specifically and highly expressed in human endothelial cells. Here, we demonstrate EC-specific expression of miR-I26 in capillaries and the larger vessels in vivo. We therefore explored the potential role of miR-126 in arteriogenesis and angiogenesis. Using miR-reporter constructs, we show that miR-126 is functionally active in EC in vitro and that it could be specifically repressed using antagomirs specifically targeting miR-I26. To study the consequences of miR-I26 silencing on vascular regeneration, mice were injected with a single dose of antagomir- 126 or a control "scramblemir" and exposed to ischemia of the left hindlimb by ligation of the femoral artery. Although miR-I26 was effectively silenced in mice treated with a single, high dose of antagomir-126, laser Doppler perfusion imaging did not show effects on blood flow recovery. In contrast, quantification of the capillary density in the gastrocnemius muscle revealed that mice treated with a high dose of antagomir- 126 had a markedly reduced angiogenic response. Aortic explant cultures of the mice confirmed the role of miR- 126 in angiogenesis. Our data demonstrate a facilitary function for miR- 126 in ischemiainduced angiogenesis and show the efficacy and specificity of antagomir-induced silencing of EC-specific microRNAs in vivo.

\section{Introduction}

Endothelial cells (EC) play an essential regulatory role in the capacity of the vasculature to adequately respond to injury or hypoxia. In arteriogenesis, EC react to elevated shear stress by recruiting and activating leukocytes that mediate remodeling of small collateral arterioles. In tissue ischemia, novel capillaries are generated by proliferation and migration of EC that sprout from pre-existing capillaries. The molecular mechanisms underlying this directive role of EC in vascular plasticity have been extensively studied and involve numerous environmental cues that elicit complex, but tightly coordinated responses in the expression of genes controlling proliferation, migration and cell-differentiation [I]. MicroRNAs constitute a recently recognized class of short, non-coding RNA molecules $(\sim 2 \mathrm{It})$ that could potentially regulate the activity of $30 \%$ of all genes at the post-transcriptional level [2].The ability of microRNAs to regulate multiple targets provides a means for coordinated control of gene expression, while also making them especially attractive candidates for regulating both cell-type specific differentiation and modulation of cell function [3].

Recently, evidence supporting a role for endothelial microRNAs in the control 
of neovascularization has been provided [4] with in vitro studies demonstrating both pro-angiogenic microRNAs (let 7b, miR-27b [5], miR-I30a [6], miR-2I0 [7]) as well as microRNAs with anti-angiogenic actions (miR-22I/222) $[8,9]$. To study the role of endothelial microRNAs in neovascularization, we recently generated an inventory of known and novel microRNAs expressed by human microvascular EC and late outgrowth EC using extensive cloning and sequencing [10]. By comparing the microRNA expression profiles of various cultured EC with other tissues and cell types, we confirmed miR-I26 to be highly abundant and specific for EC. MiR-126 is located in an intron of the epidermal growth factor-like-domain 7 gene (EGFL7) of human, mouse and zebrafish [ I I]. Due to the fact that EGFL7 expression is augmented in adult angiogenesis and vascular injury [12], we hypothesized that co-expression of miR- I 26 may also play a role in neovascularization. Recently, two papers have described a role for miR-I26 in vascular development in mice [13], and zebrafish [14]. Targeted deletion of miR- 126 resulted in vascular leakage, hemorrhaging and embryonic lethalility in a subset of the mutant mice and abnormal vessel morphology was observed in the zebrafish. In this study, we assessed the role of miR-126 in neovascularisation in the adult mouse and explored the effects of conditional silencing of this microRNA on arteriogenesis and angiogenesis in vivo. Previous research has demonstrated that intravenous injection of chemically modified and cholesterolconjugated RNA analogs (antagomirs), specifically silences complementary microRNAs in mice for up to 23 days $[15,16]$. Our studies using a mouse ischemic hind limb model demonstrate, for the first time, that antagomir-induced silencing of miR-I26 impairs ischemia-induced angiogenesis in vivo.

\section{Material and methods}

\section{Cells and cell culture}

Human embryonic kidney cells (HEK-293T), HeLa cells and immortalized mouse endothelial cells (EC), originally derived from brain capillaries (bEnd3 cells) $[28,29]$ were cultured in DMEM (Gibco/Invitrogen, Breda, The Netherlands) supplemented with pen/strep and 10\% fetal calf serum (FCS; Bio Whittaker/ Cambrex, Verviers, Belgium). Immortalized human umbilical vein EC (EC-RF24) [30]. were cultured in MI99 medium (Gibco/Invitrogen) supplemented with pen/strep, 20\% FCS, $10 \mathrm{IU} / \mathrm{mL}$ heparin (Leo Pharma, Breda, The Netherlands), 2.5\% HEPES buffer (Gibco/Invitrogen) and $12.5 \mu \mathrm{g} / \mathrm{mL}$ EC growth supplement (Sigma, St. Louis, MO). For the generation of monocyte-derived human dendritic cells (DC), human peripheral blood mononuclear cells (PBMC) were isolated from buffy coats obtained from healthy donors by Ficoll (Amersham, 's-Hertogenbosch, The Netherlands) density gradient centrifugation. Monocytes were positively selected by CDI4-MACS microbeads (Miltenyi Biotech, Bergish Gladbach, Germany) and cultured for 7 days in RPMI medium (Gibco/Invitrogen) 
containing 10\% FCS, 2\% pen/strep, human GM-CSF (5 ng/mL, Leucomax, Lelystad, The Netherlands) and human IL-4 (I0 ng/mL, Peprotech, Rocky Hill, NJ) at a density of $1.5 \times 106$ cells/ well in a 6 wells plate (Costar, Cambridge, MA). For the generation of monocyte-derived human macrophages, PBMC were cultured with RPMI medium containing $10 \%$ FCS, $2 \%$ pen/strep and human GM-CSF $(5 \mathrm{ng} / \mathrm{mL})$. Human foreskin-derived microvascular endothelial cells (hMVEC) and human umbilical vein endothelial cells (HUVEC) were isolated, cultured and characterized as described previously [3I-33].

\section{Quantification of microRNA levels}

Total RNA from different cell types and tissues was isolated using Trizol reagent (Invitrogen, Breda, The Netherlands). Expression levels of selected microRNAs were validated in triplicate by quantitative RT-PCR ( $q R T-P C R$ ). Reverse transcription was performed using a 5 minute $65^{\circ} \mathrm{C}$ incubation of $250 \mathrm{ng}$ total RNA with dNTPs (Invitrogen) and oligo(dT) (U6, Invitrogen) or using specific Taqman ${ }^{\circledR}$ microRNA probes (miR-126 and miR-423, Applied Biosystems, Nieuwerkerk a/d IJssel, The Netherlands). cDNA was synthesized using a M-MLV First-Strand Synthesis system (Invitrogen). Validation of miR-I26 and miR-423 levels was performed using Taqman $^{\circledR}$ miR assays and qRT-PCR. For normalization, a qRT$P C R$ on RNU6B was performed on CDNA obtained from the same RNA. The following primers were used for PCR: U6 (sense) CTCGCTTCGGCAGCACA and U6 (antisense) AACGCTTCACGAATTTGCGT. qRT-PCR conditions were the same as used for miR quantifications. Results were normalized using Gene Expression Analysis for iCycler IQ ${ }^{\circledR}$ RT-PCR Detection System (Bio-Rad Laboratories, Veenendaal, The Netherlands).

\section{In situ hybridizations}

In situ hybridizations were performed as essentially described [18, 34, 35]. In brief, serial paraffin-embedded sections of the human kidney were used for in situ hybridization of miR-126. The sections were rehydrated, digested with proteinase $\mathrm{K}\left(5 \mu \mathrm{g} / \mathrm{mL}, 10 \mathrm{~min}, 37^{\circ} \mathrm{C}\right)$ and post-fixated with $4 \%$ formaldehyde. The sections were hybridized overnight in hybridization mix $(50 \%$ formamide, $5 \times$ SSC, $0.1 \%$ Tween-20, $500 \mu \mathrm{g} / \mathrm{mL}$ tRNA, $0.5 \%$ citric acid $(92 \mathrm{mM})$ and $50 \mu \mathrm{g} / \mathrm{mL}$ heparin) at $53^{\circ} \mathrm{C}$ with a digoxigenin (DIG)-labeled locked nucleic acid (LNA) miR-I 26 probe ( $25 \mu \mathrm{M}$, Exiqon,Vedbaek, Denmark). The DIG-labels were visualized with sheep anti-DIG (Roche, Mannheim, Germany). MiR-I26 stained blue after overnight incubation at $50^{\circ} \mathrm{C}$ in the dark with the substrate NCT/BCIP (Roche). After staining, the sections were dehydrated in a series of ethanol and xylene. Control sections were incubated with a LNA-probe that does not bind to any known miRNAs. 


\section{MiR- I 26 reporter assays}

Cloning of one (PMIR-I26M) or four (PMIR-I26Q) target binding sites was performed into a pMIR-reportTM Expression Reporter Vector System (pMIR, Applied Biosystems), which contains an experimental firefly luciferase reporter gene. After cloning, the plasmids were sequenced to evaluate their fidelity. A renilla luciferase expressing plasmid (pRL-SV40, Promega, Leiden, The Netherlands) served as control for the efficiency of electroporation. Primer constructs used for correct cloning into PMIR-report vector were: pmiR-I26M (sense) CTAGTCAGTGGCAGCAGCATTATTACTCACGGTACGATCAGTGGCAGCA; pmiR-126M (antisense) AGCTTGCTGCCACTGATCGTACCGTGAGTAATAATGCTGCTGCCACTGA; pmiR-I26Q (sense) CTAGTAGGCGCGCCATATAGCATTATTACTCACGGTACGATATAGCATTATTACTCACGGTACGATATAGCATTATTACTCACGGTACGATATAGCATTATTACTCACGGTACGATATATAGCGCGCTACA; and pmiR-126Q (antisense) AGCTTGTAGCGCGCTATATATCGTACCGTGAGTAATAATGCTATATCGTACCGTGAG TAATAAT G CTATATCG TACCGTG A GTAATAATGCTATATCGTACCGTGAGTAATAATGCTATATGGCGCGCCTA.

\section{Design of antagomirs}

Cholesterol-conjugated RNA analogs (antagomirs, Dharmacon RNA technologies, Lafayette, $\mathrm{CO}$ ) were synthesized as previously described [15]. For antagomir- 126 the following sequence was used:5'-gscsauuauuacucacgguascsgsasChol-3'. As a control a 'scramblemir' was used, this RNA analog is constructed from a randomized nucleotide sequence which does not bind to any known microRNAs: 5'-asusgacuaucgcuauucgcsasusgs-Chol-3'. The lower case letters represent 2'-OMe-modified nucleotides; subscript 's' represents phosphorothiate linkage; 'Chol' represents a cholesterol-group linked through a hydroxyprolinol linkage (Manoharan, M., Kesavan, V., \& Rajeev, K. G. SiRNA's containing ribose substitutes to which lipophilic moieties may be attached. U.S. Pat. Appl. Publ. (2005), US 2005107325).

\section{Electroporation of EC-RF24cells}

Fibronectin-adherent EC-RF24 cells $(500.000)$ were detached by trypsin treatment and resuspended in $500 \mu \mathrm{L}$ serum free medium (Optimem; Gibco/Invitrogen) and I $\mu$ g specific PMIR-report and I 00 ng pRL-SV40 was added. The cell suspension was chilled for 10 minutes at $4^{\circ} \mathrm{C}$ and electroporated in a Gene Pulser ${ }^{\circledR}$ cuvette (Bio-Rad Laboratories) using Gene Pulser II (Bio-Rad Laboratories). After 10 minutes recovery time at room temperature, a triplicate of 150.000 cells was plated in a 24-wells plate coated with fibronectin. After 24 hours the firefly-luciferase and renilla-luciferase signals were measured using a DualLuciferase $^{\circledR}$ Assay Reporter System (Promega) in a Lumat LB9507 (EG\&G Berthold, Bundoora, Australia) and the ratio of the firefly-luciferase expression 
divided by renilla luciferase expression was calculated. Cells transfected with PMIR were taken as $100 \%$. For inhibition studies, $5 \mu \mathrm{g} / \mathrm{mL}$ antagomir- 126 or scramblemir was added on a near confluent cell layer, 16 hours prior to transfection.

\section{Hind limb ischemia model}

All animal experimental protocols were approved by the animal welfare committee of the Netherlands Organization for Applied Scientific Research (TNO). One day prior to surgery, C57BL/6 WT mice ( $n=6$ per group, age $=10$ weeks, Charles River, Maastricht, The Netherlands) were injected intravenously $(200 \mu \mathrm{L})$ with antagomir-126 at $1.0 \mathrm{mg} /$ animal (high dose, HD), $0.1 \mathrm{mg} /$ animal (low dose, LD), scramblemir at $1.0 \mathrm{mg} /$ animal (HD) or $0.1 \mathrm{mg} /$ animal (LD). Before surgery, mice were anesthetized intraperitoneally with a combination of Midazolam ( $5 \mathrm{mg} / \mathrm{kg}$, Roche), Medetomidine $(0.5 \mathrm{mg} / \mathrm{kg}$, Orion Corporation, Turku, Finland) and Fentanyl $(0.05 \mathrm{mg} / \mathrm{kg}$, Janssen Pharmaceutica, Tilburg, The Netherlands). Ischemia of the left hind limb was induced by electrocoagulation of the left common femoral artery, proximal to the bifurcation of superficial and deep femoral artery, as described [36]. The blood flow in the ligated and non-ligated hind limb was measured using laser Doppler perfusion imaging (Moor Instruments, Milwey, UK), as previously reported [37]. Measurements were performed immediately after surgery and serially up to 10 days. To control for temperature variability, animals were kept in a double-glassed vessel filled with water at constant temperature of $37^{\circ} \mathrm{C}$ for 5 minutes and during subsequent measurements. Perfusion was expressed as the ratio of left (ischemic) to right (non-ischemic) limb.

Upon sacrifice of the mice, the gastrocnemius muscle of both hind limbs was dissected and fixated overnight in $4 \%$ formaldehyde. After paraffin embedding, $4 \mu \mathrm{m}$ thick serial cross sections were made for immunohistochemical analysis. Lungs were harvested and stored at $-80^{\circ} \mathrm{C}$ for RNA analysis.

\section{Immunohistochemistry (IHC)}

Four $\mu \mathrm{m}$-thick sections of human kidney or murine gastrocnemius muscle were re-hydrated and endogenous peroxidase activity was blocked. Antigen-retrieval in the human kidney was performed by heat-treatment, the mouse gastrocnemius muscle was incubated with $10 \mathrm{mM}$ TRIS/I mM EDTA. EC were visualized with diaminobenzidine (DAB substrate KIT, Pierce, Rockford, IL) after incubation with monoclonal antibodies (moab) directed against CD3I (clone M0823 (Dako Cytomation, Eindhoven, The Netherlands) for human kidney and moab (Santa Cruz Biotechnology, Santa Cruz, CA) for the murine gastrocnemius muscle), followed by peroxidase-labeled rabbit anti-mouse-lgG (Mouse Envision Labeled Polymer horseradish peroxidase system, DakoCytomation). As negative control, primary antibodies were omitted. Sections of the human kidney were counter- 
stained with hematoxylin.

\section{Quantification of angiogenic response}

Two slides (200 $\mu \mathrm{m}$ apart) were taken of each murine gastrocnemius muscle. Several pictures were taken randomly and the percentage coverage was measured and expressed as the total area of CD3 I-positive cells per image using the Scion Imaging software (Scion Corporation, Fredrick, MD). Scramblemir (LD) was taken as $100 \%$.

\section{Aortic explants cultures}

Eleven days after antagomir-treatment, the thoracic to abdominal aorta was removed [38] and transferred to a petri dish containing MI99-medium (Gibco) supplemented with pen/strep, 20\%FSC, $10 \mathrm{lU} / \mathrm{mL}$ heparin (Leo Pharma) and $25 \mathrm{mg}$ bovine pituitary extract (Gibco). The surrounding tissue was carefully removed and the aorta was flushed several times with the same medium. The aorta was cut into small pieces and transferred to a fibronectin-coated 24 wells plate (Costar). After firm attachment of the explants (after 24 hours) extra medium was added. After 5 and 10 days in culture, pictures were taken from the explants (Leica DMI6000, Nieuw Vennep, The Netherlands) and the total surface area covered with outgrowing cells was measured in a representative area $\left(1.5 \times 10^{5} \mu \mathrm{m}^{2}\right)$.

\section{In vitro capillary formation}

The role of miR-I26 in the capacity of EC to form capillary-like structures was assessed using an in vitro angiogenesis assay kit (Chemicon, Temecula, CA) and HUVEC. HUVEC were incubated in vitro overnight with $5 \mu \mathrm{g} / \mathrm{mL}$ antagomir-126orscramblemirandadded to thematrigelAfter $8 \mathrm{~h}$,tubeformation was measured by staining the viable cells with calcein-AM $(5 \mu \mathrm{g} / \mathrm{mL}$, Molecular Probes, Leiden, The Netherlands). Total tube area and length were determined using images obtained with an inverted fluorescence microscope and the Scion Imaging software.

\section{Scratch-wound assay}

HUVEC were cultured in a 12 -wells plate (Costar) and incubated with antagomir- 126 or scramblemir ( $5 \mu \mathrm{g} / \mathrm{mL}$, 4 wells per condition, 16 hours). Next, the medium was removed and stored on ice and a scratch was made of approximately $800 \mu \mathrm{m}$ in the EC-monolayer using a pipette-tip. Cells were washed to remove cell-debris and the medium containing antagomir-126 or scramblemir was transferred back to the appropriate wells. Several images (LeicaDMI6000) were taken within 24 hours and the distance of the scratch was measured (expressed in $\mu \mathrm{m}$ ). 


\section{Statistical analysis}

Results are expressed as standard error of the mean (SEM). Statistical analysis was performed using the Mann-Whitney T-test. $\mathrm{P}<0.05$ were considered statistically significant.

\section{Results}

\section{miR-I26 is specifically expressed in endothelial cells in vitro and in vivo}

To validate the expression of miR-126 in EC, we first quantified the presence of the mature form of miR-126 in endothelial and non-endothelial cell types using a TaqMan real-time PCR assay (Figure IA). After normalization of the signals to U6 small nuclear RNA, we observed that human CD I4-derived dendritic cells (DC), human macrophages (M $\Phi)$, HeLa cells and embryonic kidney cells (HEK-293T) expressed only background levels of miR-126. As expected, both human (EC-RF24, MVEC, HUVEC) and murine (bEnd3) EC displayed over two orders of magnitude more miR-126, confirming that miR-126 is highly expressed in microvascular and macrovascular EC in vitro.

In contrast to the expression of miR-126 in cultured EC [5, 10. 17], little is known about the tissue specific expression of miR-126 in vivo. Therefore, we performed in situ hybridization analysis on human renal sections using digoxigenin-labeled locked nucleic acid (LNA) probes [18]. To localize the endothelium in these sections, we co-stained for the endothelial marker CD3 I

(A)

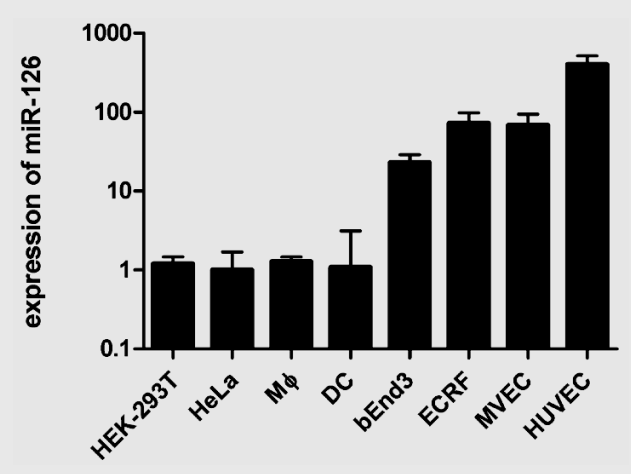

(B)

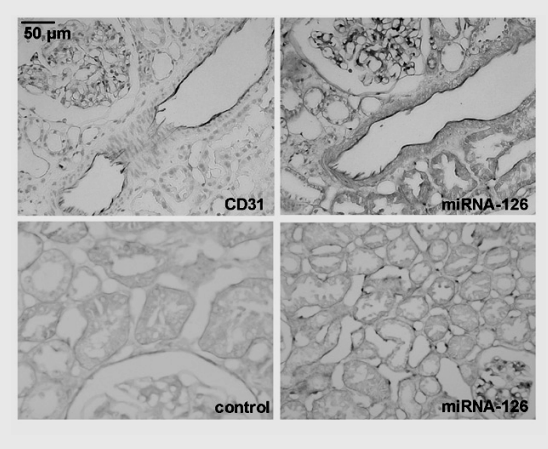

Figure I. miR- I 26 is specifically expressed in EC

(A) Total RNA was harvested from different cell types and analyzed by qRTPCR on miR-126. Obtained values were normalized by qRT-PCR on U6 snRNA. (B) Representative microscopic images of immunohistochemistry of CD3I on human renal sections and in situ hybridization of miR-I26 with LNA-probe or a control LNA-probe. Upper two pictures represent consecutive sections. 
(Figure IB). In situ hybridization of consecutive human renal sections with the LNA probe for miR-I26 demonstrated expression of miR-I26 in the CD3Ipositive endothelium of the glomerular and peritubular capillaries as well as the endothelium of the larger vessels. These findings confirm the EC-specificity of miR- 126 expression in vivo.

\section{miR- I 26 is functionally active in EC}

Next, we sought to investigate whether miR-I26 is functionally active in EC. For this, we constructed reporter plasmids containing the firefly luciferase gene under the control of the constitutive CMV promoter and no (pMIR), one (pMIRI26M) or four (PMIR-I26Q) perfect miR-I26 target sites in the 3' untranslated region of the luciferase reporter gene. Twenty four hours after electroporation of the reporter plasmids into the target cells, firefly luciferase activity was measured and normalized for electroporation efficiency using renilla luciferase activity derived from a co-electroporated expression plasmid. In HEK-293T cells, which lack miR-126 expression, luciferase expression was identical for all three reporters, indicating that the incorporation of the miR-126 target sites did not impact the luciferase transcript translation efficiency (Figure 2A).

In contrast, EC-RF24 cells displayed markedly reduced luciferase activity, with the presence of one or four miR- 126 target sites reducing luciferase expression to $13 \%(p<0.01)$ and less then $1 \%(p<0.01)$, respectively. These data clearly demonstrate a potent negative regulatory role of miR-126 in cultured EC.
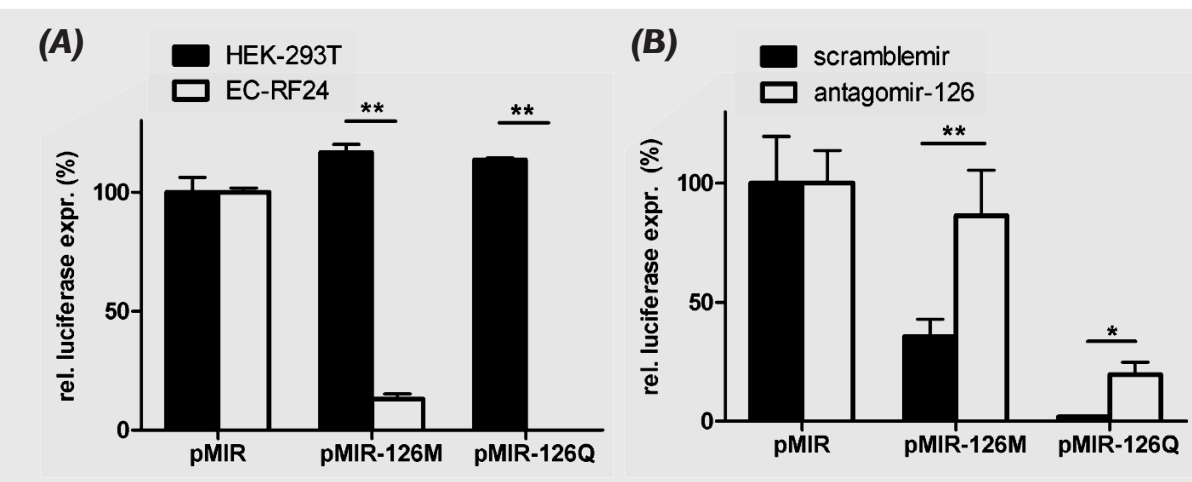

Figure 2. miR- I 26 is functionally active in vitro and can be specifically repressed by antagomir- 126 in vitro and in vivo

(A) Relative firefly luciferase expression of HEK-293T and EC-RF24 transfected with a luciferase reporter plasmid harbouring 0 (pMIR), I (pMIR-I26M) or 4 (pMIR-

126Q) perfect match target sites for miR-I26 in the $3^{\prime} U T R$ of the luciferase transcript (*** $=p<0.01$ ). (B) Relative firefly luciferase expression of transfected EC-RF24 incubated for 16 hours with $5 \mu \mathrm{g} / \mathrm{mL}$ scramblemir or antagomir- 126 $(*=p<0.05$ and $* *=p<0.01)$. 


\section{Antagomir silencing of miR- I 26 in EC}

Antagomirs have been used to specifically silence microRNAs [15], which prompted us to determine whether antagomirs can also be used to silence miR-126 in EC. Therefore, we designed both a cholesterol-conjugated modified 21 nucleotide RNA, complementary to mature miR-I 26 (antagomir-126) as well as a control RNA analog of identical composition and length, but with a random sequence (scramblemir). Using a bioinformatic approach, this sequence was chosen due to the fact that it did not match any known miR or mRNA. Addition of $5 \mu \mathrm{g} / \mathrm{mL}$ scramblemir to the culture medium of EC-RF2 4 cells, 16 hours before electroporation with the miR-1 26 reporter plasmids (Figure 2B), had little effect on the miR-I26-repressed luciferase levels of pmiR-I26M (35\%) and pmiR-I26Q (2\%). In contrast, pre-culturing the cells with $5 \mu \mathrm{g} / \mathrm{mL}$ antagomir- 126 restored luciferase levels to $86 \%(p<0.01)$ and $20 \%(p<0.05)$ for pmiR-I 26M and pmiR-I26Q, respectively. This supports the notion that antagomirs can be efficiently taken up by EC and that antagomir- 126 can be used to specifically counteract the negative regulation of target gene expression by miR-I26.

(A)

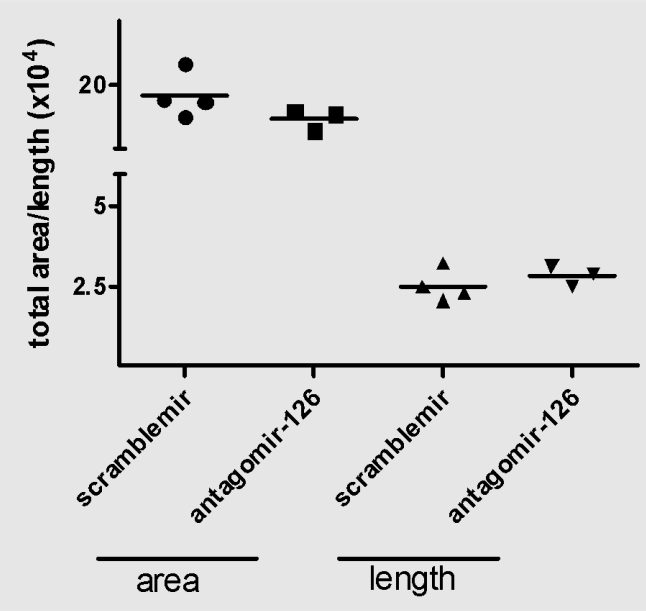

(B)
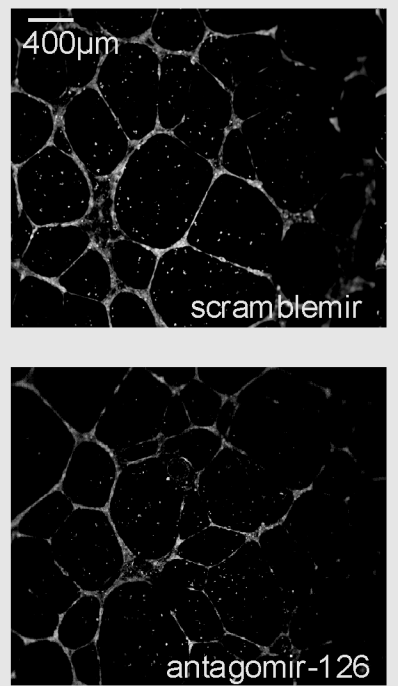

Figure 3A-B. Repression of miR- I 26 in vitro does not influence the formation of capillary structures or EC proliferation and migration

(A) Quantification of area and length of capillary structures after $8 \mathrm{~h}$ of culture on matrigel (data expressed in arbitrary units). (B) Representative microscopic images of the formation of capillary-like structures by HUVEC.

\section{Antagomir silencing of miR-I26 has no effect on in vitro angiogenesis and migration and proliferation}

To assess whether miR-I 26 plays a role in the capacity of EC to form capillarylike stuctures on matrigel, HUVEC were incubated overnight with $5 \mu \mathrm{g} / \mathrm{mL}$ 
antagomir-126 or scramblemir. Eight hours after cell seeding, tube formation was not affected by silencing of miR-I 26 (Figure 3A-B). Next, similarly-treated HUVEC were cultured to confluence and their capacity to migrate and proliferate was assessed by measuring the degree to which a $800 \mu \mathrm{m}$ scratch was re-populated by the cells in $24 \mathrm{~h}$. Again, we observed no effects of miR-126 silencing on proliferation and migration of the cells (Figure 3C-D).

(C)

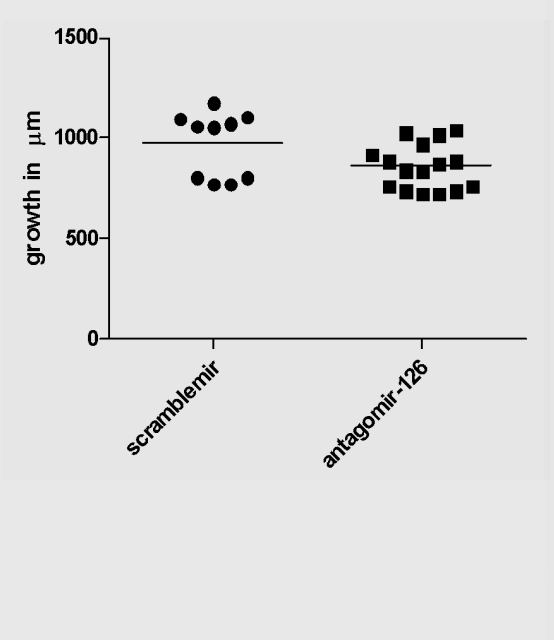

(D)

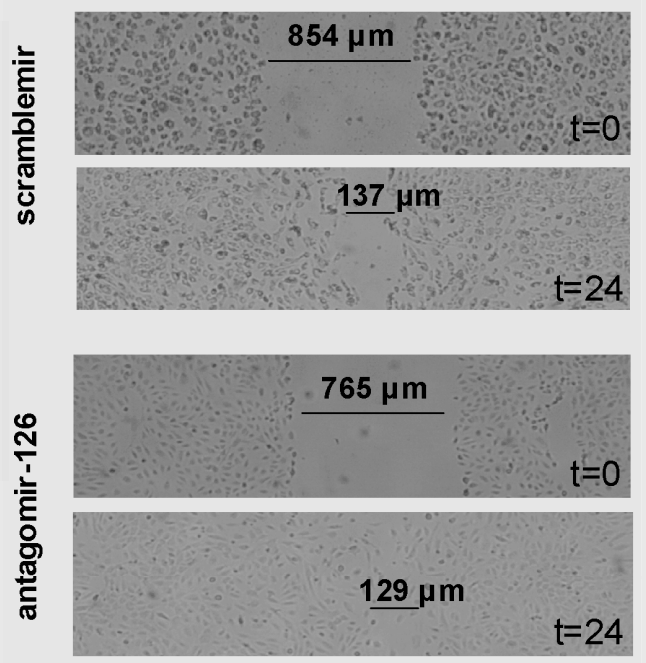

Figure 3C-D. Repression of miR-I 26 in vitro does not influence the formation of capillary structures or EC proliferation and migration

(C) Quantification of the degree of EC regrowth in a scratch-wound assay (expressed in $\mu \mathrm{m}$ from scratch border). (D) Representative microscopic images of HUVEC incubated with antagomir-126 or scramblemir before and 24h after the scratchwound assay.

\section{Antagomir silencing of miR- I 26 does not affect arteriogenesis}

The murine ischemic hindlimb model makes it possible to assess the consequences of interventions on collateral formation around the ligated femoral artery as well as on the hypoxia induced angiogenic response in the distal calf muscle [19].To investigate the role of miR-I26 in neovascularization and the use of antagomirs to silence endothelial microRNAs in vivo, we injected four groups $(n=6)$ of male C57BI/6WT mice in the tail vein with either a low dose $(0.1 \mathrm{mg}$, LD) or a high dose (1.0 mg, HD) of antagomir-126 or scramblemir. After 24 hours, unilateral hindlimb ischemia was induced by electrocoagulation of the left common femoral artery and blood flow recovery was measured over 10 days using laser Doppler perfusion imaging. As shown in Figure 4C, the progression of blood flow recovery was similar for all treated groups, reaching normal levels between day 7-I0. 
To verify that the injected antagomir- 126 had indeed silenced miR-126 in the endothelium of the mice at the end of the experiment, the lungs of the mice were harvested and the expression levels of miR- 126 and a control EC-enriched microRNA miR-423, were quantified by real-time PCR (Figure 4A-B). Whereas the mice treated with antagomir-I26 (LD) show only a marginal reduction in miR-126 expression ( 1.9 fold, $p<0.05$ versus scramblemir (LD)), mice treated

(A)

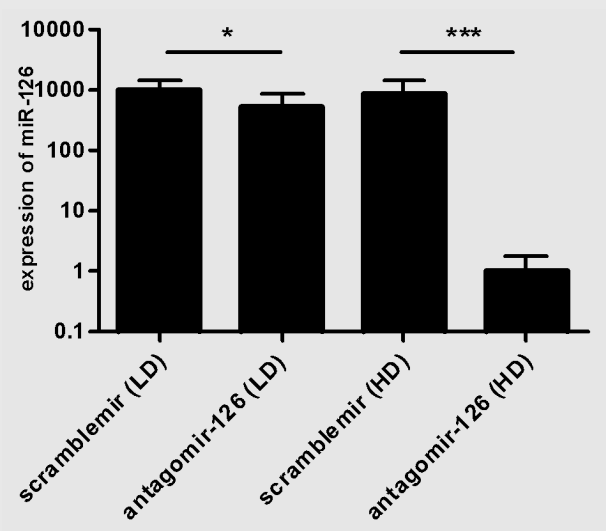

(B)

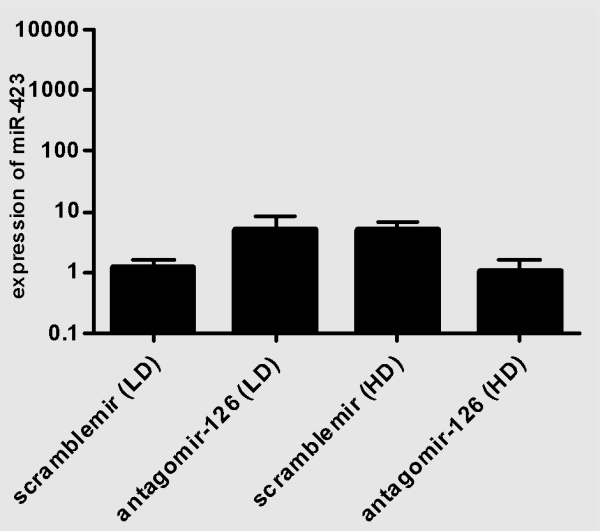

(C)

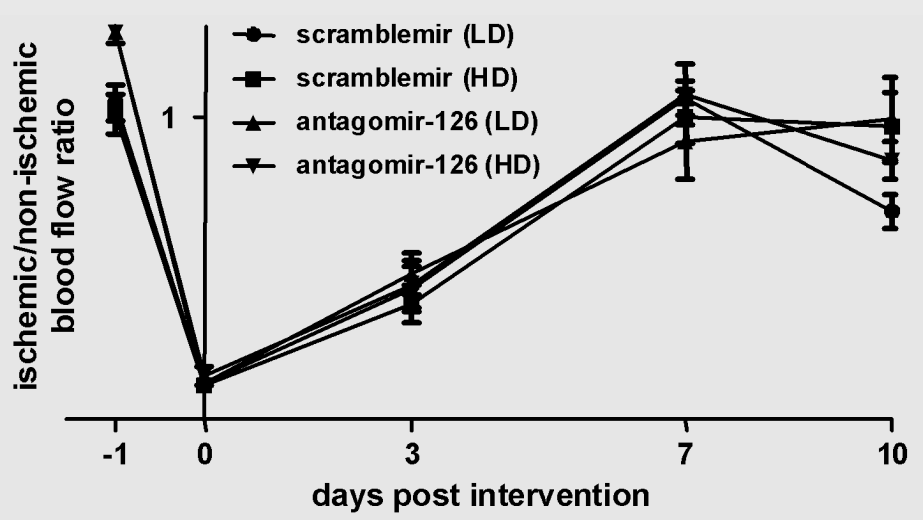

Figure 4A-C. miR-I26 silencing has no effect on collateral formation, but miR- I 26 facilitates ischemia-induced angiogenesis

(A-B) Total RNA was harvested from lungs of mice after II day treatment with high and low doses of antagomir-126 or scramblemir (LD or HD). Samples were analyzed by qRT-PCR for A, miR- 126 and B, miR-423 expression. Obtained values were normalized by qRT-PCR on U6 snRNA (* $=p<0.05$ and $* * *=p<0.001$ ). (C) Quantitative evaluation of blood flow recovery measured before and after induction of ischemia by laser Doppler perfusion imaging and expressed as a ratio of the left (ischemic) and right (non-ischemic) limb. 
with antagomir-126 (HD) displayed an over 1000-fold reduction in miR-126 expression compared to the scramblemir-treated mice $(p<0.00 \mathrm{I})$. In contrast, no significant differences were observed for the miR-423 levels in all groups. Our data demonstrate that in vivo silencing of the endothelial miR- 126 remains readily detectable ten days after administration of a single dose of $1.0 \mathrm{mg}$ of antagomir-126. Moreover, we conclude that miR-I26 is not directly involved in arteriogenesis.

\section{Antagomir silencing of miR-I26 impairs ischemia-induced angiogenesis}

To assess the effect of miR- 126 silencing on the ischemia-induced angiogenic response, we performed a detailed quantitative analysis of CD3I stained capillaries in sections of gastrocnemius muscle of all treated mice (Figure 4D-E). Mice treated with a single dose antagomir-126 (HD) showed a markedly lower density of capillary vessels compared to antagomir-126 (LD) or both control groups (35\% versus $118 \%(n=6, p<0.05), 109 \%(n=6, p<0.00 I)$ and $100 \%(n=6$, $\mathrm{p}<0.0 \mathrm{I})$ ). These studies demonstrate that silencing of miR-I26 impairs the angiogenic response to ischemia.

(D)

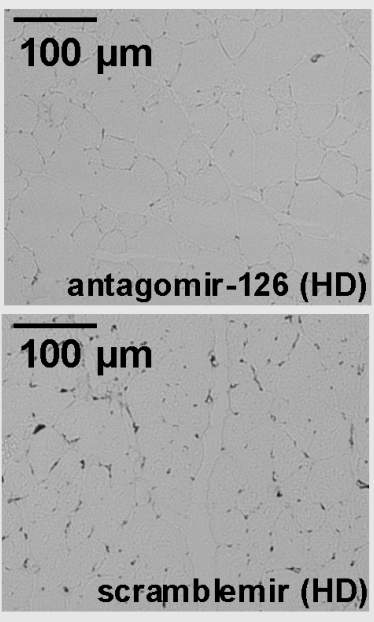

(E)

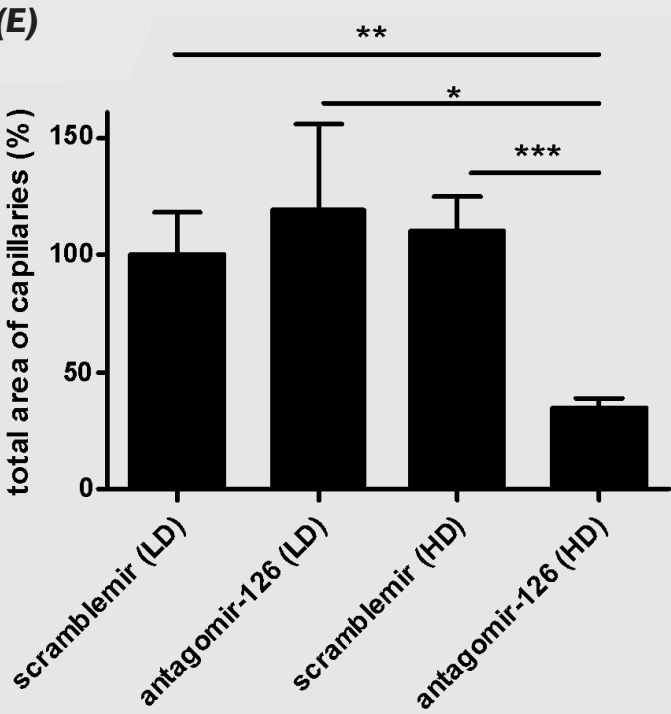

Figure 4D-E. MiR-I26 silencing has no effect on collateral formation, but miR-I 26 facilitates ischemia-induced angiogenesis

(D) Representative microscopic images of CD3I staining in ischemic murine gastrocnemius muscles. (E) Quantification of the total area of capillaries in sections of the gastrocnemius muscle 10 days after induction of ischemia $(*=p<0.05$, *** $=p<0.01$ and $* * * * p<0.001$ ). 


\section{Antagomir silencing of miR- I 26 impairs EC outgrowth in aortic ex- plant cultures}

The degree of EC outgrowth from freshly dissected pieces of abdominal aorta has been used as an assay for the potency of compounds to induce angiogenic sprouting of the aortic EC.Therefore, we cultured aortic explants on fibronectincoated plates in angiogenic medium and assessed the endothelial outgrowth after II days from mice of all four treatment groups. As shown in Figure 5, endothelial outgrowth was strongly impaired only in aortic explant cultures derived from mice treated with antagomir-126 (HD). These data support our finding of the endothelial miR- 126 is required for an appropriate angiogenic response.

(A)

(B)
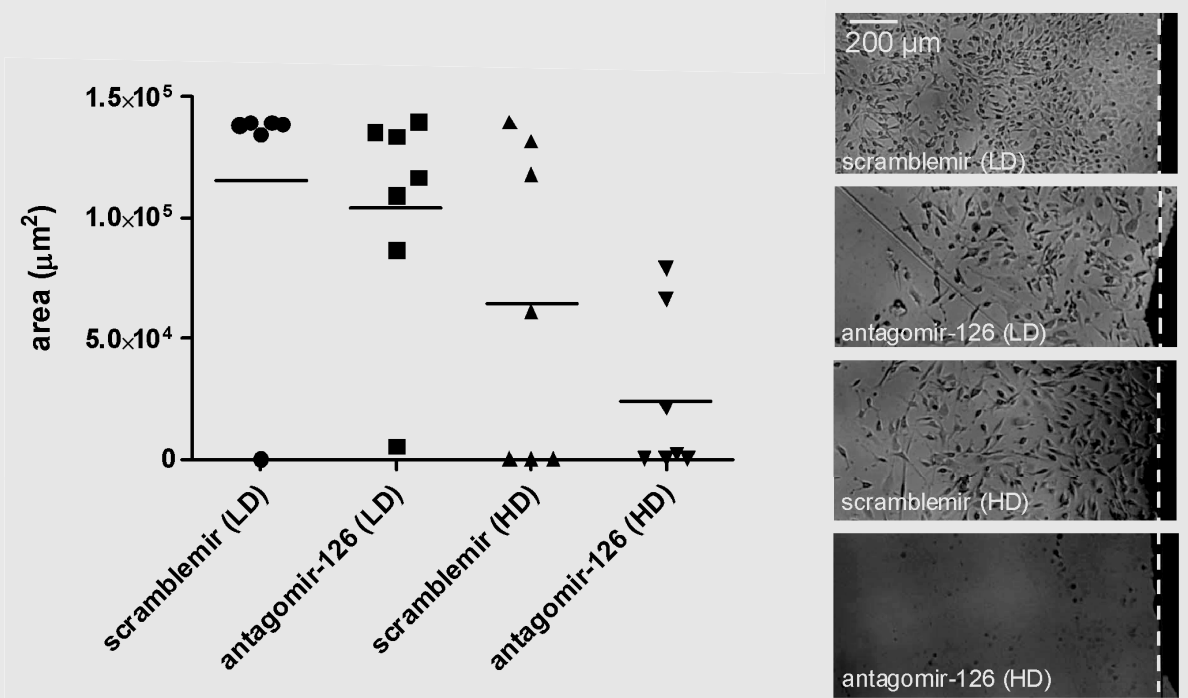

Figure 5. Repression of miR-I26 impairs endothelial outgrowth or aortic explants

(A) Quantification of total surface (in $\mu m^{2}$ ) covered by endothelial outgrowth of aortic explants. (B) Representative microscopic images of endothelial outgrowth from aortic explants.

\section{Discussion}

Previously, miR-I 26 was found to be expressed in the heart and blood vessels of zebrafish embryos [18]. We demonstrate here that miR-126 is specifically expressed in EC of capillaries and arterioles in vivo. To gain insight into a possible regulatory role for this microRNA in neovascularization, we aimed to obtain a specific miR-I26 inhibitor for conditional silencing of miR-I26 in the vascular endothelium. Recent work has resulted in the development of two potent 
approaches for the in vivo silencing of microRNAs, notably: I) Locked nucleic acid (LNA)-modified oligonucleotides for the efficient and long lasting silencing of miR-I22 function in the liver of mice and non human primates [20, 2I]. 2) Chemically modified and cholesterol-conjugated RNAs termed antagomirs for the rapid and specific degradation of microRNAs in multiple tissues after tail vein injection [15]. As cholesterol uptake is a salient feature shared by virtually all cells, including EC, we designed an antagomir directed to miR-I26. We provide evidence that a dose of $5 \mu \mathrm{g} / \mathrm{mL}$ of antagomir- 126 specifically silenced miR-I 26 function in the reporter assay in cultured EC whereas the scramblemir had little effect. Higher doses of scramblemir $(>50 \mu \mathrm{g} / \mathrm{mL})$ resulted in non-specific silencing of miR-126 (data not shown). This effect is likely the result of excessive cellular uptake of small single-stranded RNA analogs leading to non-specific interference with microRNA repression. These results emphasize the need for equally dosed scramblemir controls, for studies assessing microRNA silencing. This may be particularly relevant for in vivo studies where there is less control over the distribution of antagomirs over the different tissues.

In mice treated with antagomir-126, we validated the specificity of miR-126 silencing by quantifying the level of mature miR-I26 in total lung tissue. This was based on previous observations that, of all organs profiled for microRNAexpression by extensive cloning and sequencing, the lung displays the highest levels of miR-126 expression [13, 22] (e.g. 3 fold higher than skin, 8 fold higher than in heart and 130 fold higher than in total brain tissue [10]). We observed that 10 days after administration of a single, $1.0 \mathrm{mg}$ injection of antagomir- 126 per mouse, was sufficient to almost completely abrogate miR-I 26 expression in lung tissue, whereas miR-126 remained readily detectable in the low dose group as well as the control scramblemir groups. As a single injection of $1.0 \mathrm{mg}$ is low compared to the reported dose needed for silencing of the liver specific miR-I22 (3 consecutive injections of $2 \mathrm{mg}$ per mouse), we conclude that EC readily take up antagomirs from the circulation and may therefore be highly useful for studying endothelial microRNA function in vivo.

Leukocyte recruitment by the endothelium also plays a critical role in arteriogenesis [I]. Recently, it has been reported that vascular cell adhesion molecule I (VCAM-I) is a target for miR-I26 in HUVEC and that decreasing miR-I26 levels increased the adherence of leukocytes in vitro [23]. However, we did not observe any differences in blood flow recovery after femoral artery ligation in either of the treated groups. Therefore, we conclude that miR- 126 regulation of VCAM-I expression probably is likely not a rate-limiting factor for in vivo arteriogenesis.

Our data do support, however, a role for miR-126 in the angiogenic response. The reduction of tissue miR-I26 expression in the high dose treated mice is associated with a reduction in capillary density in gastrocnemius muscle compared to the scramblemir-treated mice. Likewise, we observed impaired out- 
growth of EC from aortic sections of miR-126-silenced mice.

Surprisingly, in vitro experiments designed to assess the relatively short term effects of antagomir-126 silencing in HUVEC revealed no differences in the formation of capillary-like structures, or cellular migration and proliferation (scratch/wound assay). This observation is compatible with the notion that different mechanisms are required for 3-dimensional sprouting of EC into a matrix compared to 2-dimensional cell movement in a culture dish [24]. The effects of miR-I26 on angiogenesis most likely involve mechanisms operational in EC in the in vivo context that involves the interaction with pericytes, EC matrix and the basement membrane. For instance, physical contact between EC and pericytes is thought to induce a quiescent, non-sprouting phenotype [25]. Initiation of angiogenic sprouting is preceded by the formation of so-called tip-cells that lead the sprouting, while the trailing EC must maintain their connection to the patent vasculature [26].Tip-cell formation in both mice and zebrafish is regulated by Notch signaling pathways and vascular endothelial cell growth factor receptor-3 (VEGFR-3) is involved in the generation of tip-cells. In mice, it has been established that the Sprouty-related Ena/VASP homology I domain containing protein (Spred-I) can function as a potent repressor of VEGFR-3 [26]. Spred-I and phosphoinositol-3 kinase regulatory subunit 2 (PIK3R2, p85- $\beta$ ), another protein actively involved in the negative regulation of VEGF signaling [27], are both predicted targets of miR-126 (http://www.targetscan.org). Consequently, upregulation of miR- 126 would thus facilitate angiogenesis by reducing the expression of both repressors of VEGF signaling whereas low levels of miR-I26 would be associated with elevation of Spred-I or PIK3R2and repress angiogenic signaling. Indeed, two recent studies reported that targeted deletion of miR- 126 in mice and zebrafish impairs angiogenesis likely through dysregulation of Spred-I and PIK3R2 expression suggesting a critical role for this microRNA in angiogenic signaling events during embryogenesis $[13,14]$. Here we demonstrate that miR- 126 also plays a key role in the regulation of ischemia-induced angiogenesis in adult mice. Using qRT-PCR we observed that antagomir-mediated silencing of miR-I 26 in murine endothelial cells (bEnd3) led to a concomitant four fold upregulation of Spred-I mRNA (data not shown). Therefore, it is tempting to speculate that upregulation of Spred-I and PIK3R2 levels also underlies the anti-angiogenic effects observed in our study.

Taken together, we have demonstrated that functional activity of miR-I26 is required for the ischemia-induced angiogenic response in vivo. In addition, our study supports the potential therapeutic use of antagomir-based approaches for conditional silencing of microRNAs in the endothelium in vivo.

\section{Sources of funding}

CvS and HdB are supported by grants (2006BI45 and 2005B 106 respectively) from the Netherlands Heart Foundation, The Hague. RB is supported by a grant 
(C07.2227) from the Dutch Kidney Foundation. AJvZ is supported by a grant from the Genzyme Renal Innovations Program.

\section{References}

I. Adams RH, Alitalo K. Molecular regulation of angiogenesis and lymphangiogenesis. Nat Rev Mol Cell Biol 2007; 8: 464-78.

2. Filipowicz W, Bhattacharyya SN, Sonenberg N. Mechanisms of post-transcriptional regulation by microRNAs: are the answers in sight? Nat Rev Genet 2008; 9:102-I4.

3. Xie X, Lu J, Kulbokas EJ, Golub TR, Mootha V, Lindblad-Toh K, Lander ES, Kellis M. Systematic discovery of regulatory motifs in human promoters and 3' UTRs by comparison of several mammals. Nature; 434: 338-45.

4. Urbich C, Kuehbacher A, Dimmeler S. Role of microRNAs in vascular diseases, inflammation, and angiogenesis. Cardiovasc Res 2008.

5. Kuehbacher A, Urbich C, Zeiher AM, Dimmeler S. Role of Dicer and Drosha for endothelial microRNA expression and angiogenesis. Circ Res 2007;101: 59-68.

6. Chen Y, Gorski DH. Regulation of angiogenesis through a microRNA miR-130a. that downregulates antiangiogenic homeobox genes GAX and HOXA5. Blood 2008; I I : 1217-26.

7. Fasanaro P, D’Alessandra Y, Di Stefano V, Melchionna R, Romani S, Pompilio G, Capogrossi MC, Martelli F. MicroRNA-2IO modulates endothelial cell response to hypoxia and inhibits the receptor tyrosine kinase ligand Ephrin-A3.J Biol Chem 2008; 283: I5878-83.

8. Poliseno L, Tuccoli A, Mariani L, Evangelista M, Citti L, Woods K, Mercatanti A, Hammond S, Rainaldi G. MicroRNAs modulate the angiogenic properties of HUVECs. Blood 2006; 108 : 3068-7I.

9. Suarez Y, Fernandez-Hernando C, Pober JS, Sessa WC. Dicer dependent microRNAs regulate gene expression and functions in human endothelial cells. Circ Res 2007; 1008.: I I64-73.

10. Berezikov E, van Tetering G, Verheul M, van de Belt J, van Laake L, Vos J, Verloop R, van de Wetering M, Guryev V,Takada S, van Zonneveld AJ, Mano H, Plasterk R, Cuppen E. Many novel mammalian microRNA candidates identified by extensive cloning and RAKE analysis. Genome Res 2006; 16: 1289-98.

II. Rodriguez A, Griffiths-Jones S, Ashurst JL, Bradley A. Identification of mammalian microRNA host genes and transcription units. Genome Res 2004; I4: I902- 10.

12. Campagnolo L, Leahy A, Chitnis S, Koschnick S, Fitch MJ, Fallon JT, Loskutoff D, Taubman MB, Stuhlmann H. EGFL7 is a chemoattractant for endothelial cells and is up-regulated in angiogenesis and arterial injury. Am J Pathol 2005; 167: 275-84.

13. Wang S,Aurora AB, Johnson BA, Qi X, McAnally J, Hill JA, Richardson JA, Bassel-Duby R, Olson EN. The endothelial-specific microRNA miR-126 governs vascular integrity and angiogenesis. Dev Cell 2008; 15: 261-7I.

14. Fish JE, Santoro MM, Morton SU, Yu S, Yeh RF, Wythe JD, Ivey KN, Bruneau BG, Stainier DY, Srivastava D. MiR-I 26 regulates angiogenic signaling and vascular integrity. Dev Cell 2008; I5: 272-84.

15. Krutzfeldt J, Rajewsky N, Braich R, Rajeev KG, Tuschl T, Manoharan M, Stoffel M. Silencing of microRNAs in vivo with 'antagomirs'. Nature 2005; 438: 685-9.

16. Krutzfeldt J, Kuwajima S, Braich R, Rajeev KG, Pena J,TuschI T, Manoharan M, Stoffel M. Specificity, duplex degradation and subcellular localization of antagomirs. Nucleic Acids Res 2007; 35 : 2885-92.

17. Wienholds E, Plasterk RH. MicroRNA function in animal development. FEBS Lett 2005; 579: $59 \mid 1-22$.

18. Wienholds E, Kloosterman WP, Miska E, Alvarez-Saavedra E, Berezikov E, de Bruijn E, Horvitz HR, Kauppinen S, Plasterk RH. MicroRNA expression in zebrafish embryonic development. Science 2005; 309: 310-I.

19. Madeddu P, Emanueli C, Spillmann F, Meloni M, Bouby N, Richer C, henc-Gelas F, van Weel 
V, Eefting D, Quax PH, Hu Y, Xu Q, Hemdahl AL, van Golde J, Huijberts M, de Lussanet Q, Struijker-Boudier H, Couffinhal T, Duplaa C, Chimenti S, Staszewsky L, Latini R, Baumans V, Levy BI. Murine models of myocardial and limb ischemia: diagnostic end-points and relevance to clinical problems. Vascul Pharmacol 2006; 45: 28I-30I.

20. Elmen J, Lindow M, Schutz S, Lawrence M, Petri A, Obad S, Lindholm M, Hedtjarn M, Hansen HF, Berger U, Gullans S, Kearney P, Sarnow P, Straarup EM, Kauppinen S. LNA-mediated microRNA silencing in non-human primates. Nature 2008; 452: 896-9.

21. Elmen J, Lindow M, Silahtaroglu A, Bak M, Christensen M, Lind-Thomsen A, Hedtjarn M, Hansen JB, Hansen HF, Straarup EM, McCullagh K, Kearney P, Kauppinen S. Antagonism of microRNA- 122 in mice by systemically administered LNA-antimiR leads to up-regulation of a large set of predicted target mRNAs in the liver. Nucleic Acids Res 2008; 36: I I 53-62.

22. Lagos-Quintana M, Rauhut R, Yalcin A, Meyer J, Lendeckel W, Tuschl T. Identification of tissuespecific microRNAs from mouse. Curr Biol 2002 April 30; 12 : 735-9.

23. Harris TA, Yamakuchi M, Ferlito M, Mendell JT, Lowenstein CJ. MicroRNA- 26 regulates endothelial expression of vascular cell adhesion molecule I. Proc Natl Acad Sci U SA 2008; 105: I5|6-2|.

24. Diehl F, Rossig L, Zeiher AM, Dimmeler S, Urbich C. The histone methyltransferase MLL is an upstream regulator of endothelial-cell sprout formation. Blood 2007; 109: 1472-8.

25. Armulik A, Abramsson A, Betsholtz C. Endothelial/pericyte interactions. Circ Res 2005; 97: 5I2-23.

26. Siekmann AF, Covassin L, Lawson ND. Modulation of VEGF signalling output by the Notch pathway. Bioessays 2008; 30: 303-I3.

27. Jiang BH, Zheng JZ,Aoki M,Vogt PK. Phosphatidylinositol 3-kinase signaling mediates angiogenesis and expression of vascular endothelial growth factor in endothelial cells. Proc Natl Acad Sci U SA 2000; 97: 1749-53.

28. Williams RL, Courtneidge SA, Wagner EF. Embryonic lethalities and endothelial tumors in chimeric mice expressing polyoma virus middle T oncogene. Cell 1988; 52: I21-31.

29. Williams RL, Risau W, Zerwes HG, Drexler H, Aguzzi A, Wagner EF. Endothelioma cells expressing the polyoma middle $T$ oncogene induce hemangiomas by host cell recruitment. Cell 1989; 57:1053-63.

30. Fontijn R, Hop C, Brinkman HJ, Slater R, Westerveld A, van Mourik JA, Pannekoek H. Maintenance of vascular endothelial cell-specific properties after immortalization with an amphotrophic replication-deficient retrovirus containing human papilloma virus 16 E6/E7 DNA. Exp Cell Res 1995; 216:199-207.

31. Defilippi P, van Hinsbergh V, Bertolotto A, Rossino P, Silengo L, Tarone G. Differential distribution and modulation of expression of alpha I/beta I integrin on human endothelial cells.J Cell Biol 199I; I 14:855-63.

32. van Hinsbergh V, Binnema D, Scheffer MA, Sprengers ED, Kooistra T, Rijken DC. Production of plasminogen activators and inhibitor by serially propagated endothelial cells from adult human blood vessels. Arteriosclerosis 1987; 7: 389-400.

33. Jaffe EA, Nachman RL, Becker CG, Minick CR. Culture of human endothelial cells derived from umbilical veins. Identification by morphologic and immunologic criteria. J Clin Invest 1973; 52: 2745-56.

34. de Boer W sl, Sont JK, van Schadewijk A, Stolk J, van Krieken JH, Hiemstra PS. Monocyte chemoattractant protein I, interleukin 8, and chronic airways inflammation in COPD.J Pathol 2000l; 190:619-26.

35. Thisse B, Heyer V, Lux A, Alunni V, Degrave A, Seiliez I, Kirchner J, Parkhill JP, Thisse C. Spatial and temporal expression of the zebrafish genome by large-scale in situ hybridization screening. Methods Cell Biol 2004; 77:505-19.

36. van Weel V, Deckers MM, Grimbergen JM, van Leuven KJ, Lardenoye JH, Schlingemann RO, van Nieuw Amerongen GP, van Bockel JH, van Hinsbergh V, Quax PH.Vascular endothelial growth 
factor overexpression in ischemic skeletal muscle enhances myoglobin expression in vivo. Circ Res 2004; 95: 58-66.

37. Couffinhal T, Silver M, Zheng LP, Kearney M,Witzenbichler B, Isner JM. Mouse model of angiogenesis. Am J Pathol I998; I52: I667-79.

38. Blacher S, Devy L, Burbridge MF, Roland G, Tucker G, Noel A, Foidart JM. Improved quantification of angiogenesis in the rat aortic ring assay. Angiogenesis 2001 ; 4: I33-42. 
